\title{
Lyme disease in a Brazilian traveler who returned from Germany ${ }^{\star}$
}

\author{
Lívia Montelo Araújo Jorge ${ }^{1}$ \\ Adriana Rego Hozannah ${ }^{1}$
}

\author{
Omar Lupi ${ }^{1}$ \\ Fred Bernardes Filho ${ }^{2}$
}

DOI: http:/ / dx.doi.org/10.1590/abd1806-4841.20175584

\section{Dear Editor}

Lyme disease (LD) is a multisystem infectious disease with prominent cutaneous findings. It is caused by spirochetes belonging to the Borrelia burgdorferi sensu lato complex, mainly transmitted by the bite of infected Ixodes ticks. ${ }^{1,2}$ In Europe, it is endemic in Germany, Austria, Denmark, and Sweden; in the Americas, it is considered a public health problem in the United States. ${ }^{1}$ As a notifiable disease, cases of borreliosis have been reported in Brazil in the states of Amazonas, Tocantins, Espírito Santo, Mato Grosso do Sul, Rio de Janeiro, and São Paulo. ${ }^{2}$

The purpose of this article is to describe the occurrence of LD in a Brazilian tourist who returned from Germany, and to alert the importance of early detection of the disease.

A 40-year old female patient, resident in Rio de Janeiro, sought our dermatology service after noticing an erythematous patch with centrifugal growth and three weeks of evolution on the left thigh. She denied previous treatment or comorbidities and had no systemic symptoms. Two months before the onset of the lesion, the patient had returned from a trip to Germany, where she was camping in a rural area and suffered a tick bite on the left thigh. She reported having noticed the tick attached to her thigh in the morning, but was unable to tell the length the tick had been feeding. She removed the tick by pinching it with her fingernails. Dermatological examination revealed an erythematous, infiltrated lesion on the inner side of the left thigh, with edge and center presenting a more intense red color (Figure 1). We suggested the hypothesis of $\mathrm{LD}$, given the patient's recent history of travel to a borreliosis-endemic country, which was confirmed with positive serology for B. burgdorferi (IgM). VDRL, FTA-Abs, and FAN research were negative. Histopathological examination after lesion biopsy revealed perivascular inflammatory infiltrate, predominantly lymphocytic, with plasma cells in the superficial and middle dermis (Figure 2). Warthin-Starry staining was negative. The patient was treated with tetracycline $(250 \mathrm{mg})$ orally every six hours for 14 days with thigh lesion regression (Figure 3).

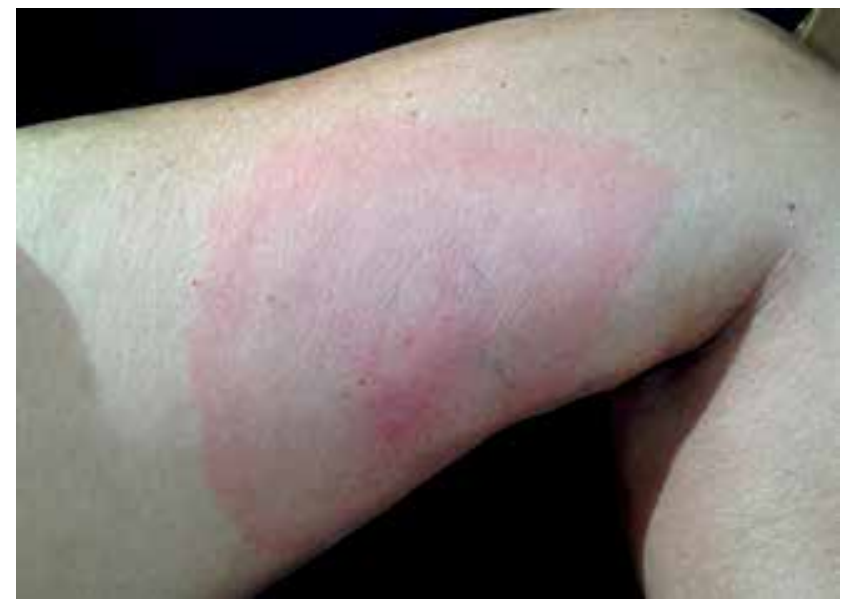

FIGURE 1: Bull's eye erythematous infiltrated legion on the inner side of the left thigh

LD's clinical picture can be divided into three stages: the first stage is characterized by predominantly cutaneous lesions, and its main manifestation is the migratory erythema, reported in $60 \%$ $83 \%$ of cases; the second stage can occur with articular, neurological, cardiac, and ophthalmologic manifestations; the third stage features chronic rheumatologic, neurological, ophthalmological, and cutaneous pictures. ${ }^{1,2}$

Diagnosis is based on epidemiological, clinical, and laboratorial aspects. ${ }^{1,2}$ Histological findings by hematoxylin-eosin staining of the present case are suggestive for borreliosis. Failure to demonstrate the presence of B. burgdorferi using silver staining is justified by the low sensitivity of this technique, which varies from $10 \%-40 \% .^{2}$ In Brazil, due to the impossibility to identify the etiologic agent of LD - because of the difference between etiologic agent and vectors, and the lack of standardization of laboratory methods - cases be-

Received on 15.01.2016

Approved by the Advisory Board and accepted for publication on 10.07.2016

* Work performed at Policlínica Geral do Rio de Janeiro (PGRJ) - Rio de Janeiro (RJ), Brazil.

Financial Support: None.

Conflict of Interest: None.

Dermatology Service of Policlínica Geral do Rio de Janeiro (PGRJ) - Rio de Janeiro (RJ), Brazil.

Division of Dermatology, Department of Clinical Medicine of Ribeirão Preto Medical School, Universidade de São Paulo (USP) - Ribeirão Preto (SP), Brazil.

(C)2017 by Anais Brasileiros de Dermatologia 

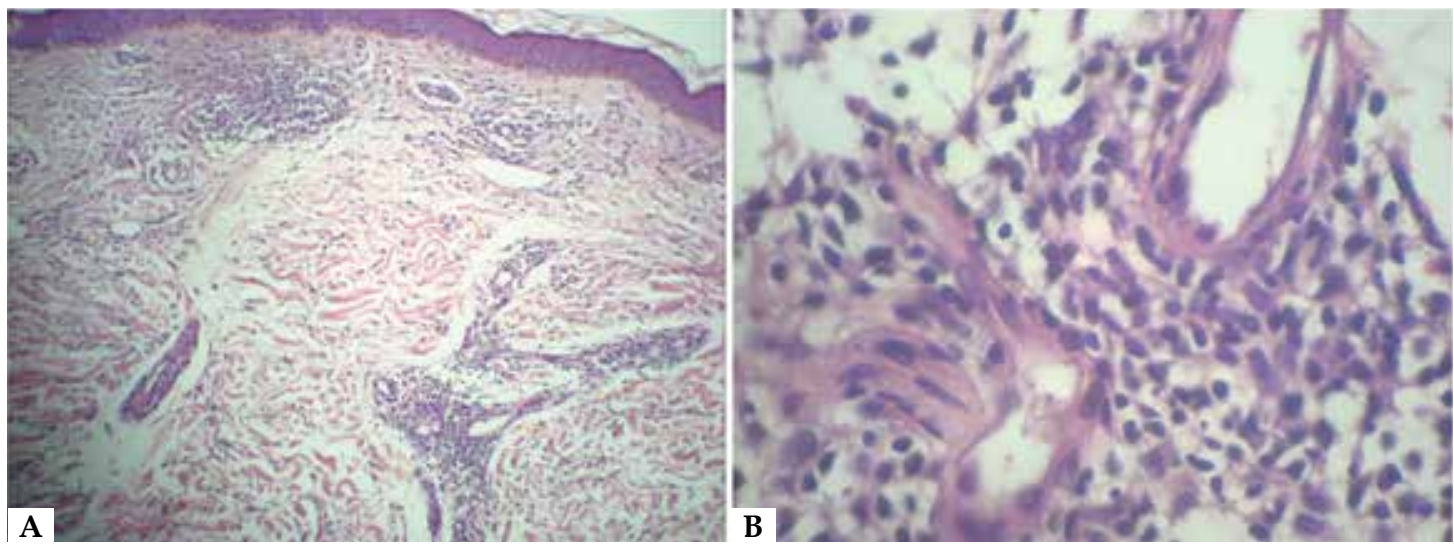

FIGURE 2:

Perivascular inflammatory infiltrate, predominantly lymphocytic with plasma cells in the superficial and middle dermis (A: Hematoxylin \& eosin x40; B: Hematoxylin $\& \operatorname{eosin} \times 400)$

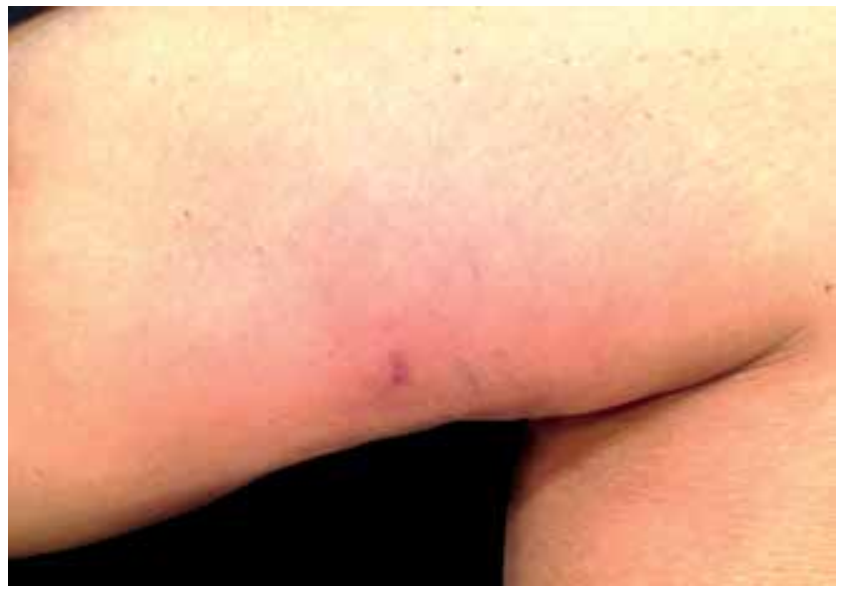

FIGURE 3: Regression of the lesion after the end of treatment

gan to be called borreliosis-simile or Baggio-Yoshinari syndrome, caused by spirochetes of the B. burgdorferi sensu lato complex. They have atypical morphology, are uncultivable and transmitted by ticks of the genus Amblyomma and Rhipicephalus. ${ }^{2}$

However, Santos et al. ${ }^{3}$ provided clinical and serological evidences that LD is indeed found in Brazil and that there is infection by Borrelia sp. in the Brazilian Amazon. In their study, 7\% of the 270 serum samples evaluated contained specific antibody to C6 an-

\section{REFERENCES}

1. Santos M, Haddad Júnior V, Ribeiro-Rodrigues R, Talhari S. Lyme borreliosis. An Bras Dermatol. 2010;85:930-8.

2. Carranza-Tamayo CO, Costa JN, Bastos WM. Lyme disease in the state of Tocantins, Brazil: report of the first cases. Braz J Infect Dis. 2012;16:586-9.

3. Santos M, Ribeiro-Rodrigues R, Lobo R, Talhari S. Antibody reactivity to Borrelia burgdorferi sensu stricto antigens in patients from the Brazilian Amazon region with skin diseases not related to Lyme disease. Int J Dermatol. 2010;49:552-6.

4. Talhari S, de Souza Santos MN, Talhari C, de Lima Ferreira LC, Silva RM Jr, Zelger $B$, et al. Borrelia Burgdorferi "sensu lato" in Brazil: Occurrence confirmed by immunohistochemistry and focus floating microscopy. Acta Trop. 2010;115:200-4.

5. Zimmerman RF, Belanger ES, Pfeiffer CD. Skin infections in returned travelers: an update. Curr Infect Dis Rep. 2015;17:467. tigen of B. burgdorferi; 6 (46.2\%) out of 19 ELISA-positive patients showed positive Western blot reactions for Borrelia antigen. ${ }^{3}$ This observed frequency coincided with the data of a study Cotia (7.5\%) and high risk areas in North America. ${ }^{3,4}$ Talhari et al. ${ }^{4}$ demonstrated - with the association of specific immunohistochemical techniques and focus floating microscopy - the presence of B. burgdorferi in $22.7 \%$ of patients with clinical and histopathological evidences suggestive of multiple sclerosis in the metropolitan region of Manaus, strengthening the evidence that LD is found in the Brazilian Amazon region. ${ }^{4}$

With the increase in international travel in the past decades, a greater variety of infectious diseases has been observed. Many infections previously considered uncommon in travelers have been described and may confuse professionals who are not familiar with the new clinical and epidemiological pictures. ${ }^{5}$ It is key to advise those who travel to borreliosis-endemic areas to avoid insect bites, especially ticks, by using long sleeve clothes and repellents.

We emphasize that early diagnosis of borreliosis is imperative, for the delay in its detection may result in serious health problems - such as atrioventricular block, ventricular repolarization disorders, encephalitis, myelitis, among others - with non-reversible consequences despite treatment.]

Acknowledgments to colleagues Dr. Laila Klotz de Almeida Balassiano and Dr. Isabela Tavares de Figueiredo.
MAILING ADDRESS:
Livia Montelo Araújo Jorge
Avenida Deputado Jamel Cecílio, 2496, sala B23, edifício New Business Style, Jardim Goiás.
74810-100 - Goiânia - GO
Brazil
E-mail:draliviamontelo@yahoo.com.br

How to cite this article: Jorge LMA, Lupi O, Hozannah AR, Bernardes Filho F. Lyme disease in a Brazilian traveler who returned from Germany. An Bras Dermatol. 2017;92(1):148-9. 